\title{
Secure Multi-party Computation for Inter-organizational Process Mining
}

\author{
Gamal Elkoumy ${ }^{1}$, Stephan A. Fahrenkrog-Petersen ${ }^{2}$, Marlon Dumas ${ }^{1(\bowtie)}$, \\ Peeter Laud ${ }^{3}$, Alisa Pankova ${ }^{3}$, and Matthias Weidlich ${ }^{2}$ \\ 1 University of Tartu, Tartu, Estonia \\ \{gamal.elkoumy, marlon.dumas\}@ut.ee \\ ${ }^{2}$ Humboldt-Universität zu Berlin, Berlin, Germany \\ $\{$ fahrenks, weidlima\}@hu-berlin.de \\ 3 Cybernetica, Tartu, Estonia \\ \{peeter.laud, alisa.pankova\}@cyber.ee
}

\begin{abstract}
Process mining is a family of techniques for analyzing business processes based on event logs extracted from information systems. Mainstream process mining tools are designed for intra-organizational settings, insofar as they assume that an event log is available for processing as a whole. The use of such tools for inter-organizational process analysis is hampered by the fact that such processes involve independent parties who are unwilling to, or sometimes legally prevented from, sharing detailed event logs with each other. In this setting, this paper proposes an approach for constructing and querying a common artifact used for process mining, namely the frequency and time-annotated DirectlyFollows Graph (DFG), over multiple event logs belonging to different parties, in such a way that the parties do not share the event logs with each other. The proposal leverages an existing platform for secure multiparty computation, namely Sharemind. Since a direct implementation of DFG construction in Sharemind suffers from scalability issues, we propose to rely on vectorization of event logs and to employ a divide-andconquer scheme for parallel processing of sub-logs. The paper reports on experiments that evaluate the scalability of the approach on real-life logs.
\end{abstract}

Keywords: Process mining $\cdot$ Privacy $\cdot$ Secure multi-party computation

\section{Introduction}

Contemporary process mining techniques enable users to analyze business processes based on event logs extracted from information systems [1]. The outputs of process mining techniques can be used, for example, to identify performance bottlenecks, waste, or compliance violations. Existing process mining techniques require access to the entire event log of a business process. Usually, this requirement can be fulfilled when the event log is collected from one

(C) Springer Nature Switzerland AG 2020

S. Nurcan et al. (Eds.): BPMDS 2020/EMMSAD 2020, LNBIP 387, pp. 166-181, 2020.

https://doi.org/10.1007/978-3-030-49418-6_11 
or multiple systems within the same organization. In practice, though, many business processes involve multiple independent organizations. We call such processes inter-organizational business processes. An example of such process is the process for ground handling of an aircraft, as illustrated in Fig. 1. This process involves two parties: the airline and the ground handler (called "airport" in the model).

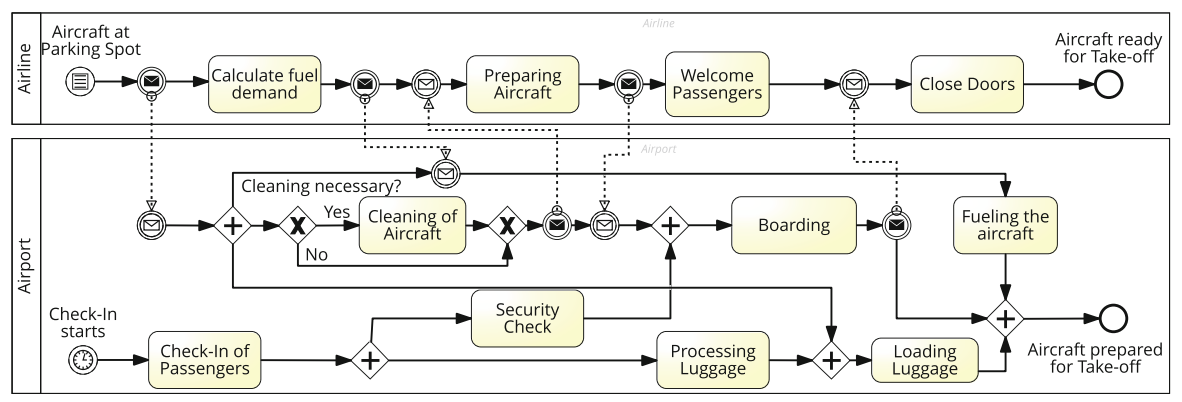

Fig. 1. Aircraft ground handling process.

Due to confidentiality concerns as well as privacy regulations, such as GDPR ${ }^{1}$ and HIPAA $^{2}$, it is not always possible for organizations to share process execution data with each other. Exchanging execution data may reveal personal information of customers or it may expose business secrets. As a result, common techniques for process mining cannot be employed for inter-organizational business processes. Yet, analyzing these processes is often crucial for improving operational performance. With reference to the above scenario, the effective coordination of ground handling activities is crucial for both involved parties. It determines the number of flights an airport can operate and the number of flights an airline can offer. At the same time, each of the parties needs to protect their confidential data.

In this paper, we focus on the question of how to enable process mining for inter-organizational business processes without requiring the involved parties to share their private event logs or trust a third party. To this end, we propose an architecture for process mining based on secure multi-party computation (MPC) [25]. In essence, MPC aims at the realization of some computation over data from multiple parties, while exposing only the result of the computation, but keeping the input data private. We consider the setting of an MPC platform where the involved parties upload their event logs to a network of compute nodes. Before the upload, secret sharing algorithms locally split each single data value into different parts (i.e., shares) that are then stored at different nodes. Since each share does not provide any information about the original

\footnotetext{
1 https://eur-lex.europa.eu/eli/reg/2016/679/oj.

2 https://www.hhs.gov/hipaa/.
} 
data, the uploaded event log is encrypted and exposed neither to the platform operator nor other involved parties. Nonetheless, the MPC platform enables the computation over the encrypted data through protocols for result sharing among the nodes.

We realize the above architecture to answer analysis queries that are common in process mining. Specifically, we show how to construct a frequency and timeannotated Directly-Follows Graph (DFG), which is a starting point for process discovery algorithms and performance analysis. While keeping the computed DFG private, we are revealing only the output of performance analysis queries such as finding the top-k bottlenecks (i.e. activities with longer cycle time) or the top-k most frequent hand-offs. We implement our proposed architecture using the Sharemind platform [7]. In order to tackle scalability issues that would be imposed by a naive implementation, we employ vectorization of event logs and propose a divide-and-conquer scheme for parallel processing of sub-logs. We test the effectiveness of these optimizations in experiments with real-world event logs.

The remainder of the paper is structured as follows. Section 2 lays out related work and the background for this work. Section 3 introduces our architecture for privacy-preserving inter-organizational process mining along with the optimizations needed for efficient implementation. An experimental evaluation is presented in Sect. 4 , before Sect. 5 concludes the paper.

\section{Background and Related Work}

In this section, we review work on privacy-preserving process mining, interorganizational process mining, and secure multi-party computation.

\subsection{Privacy-Preserving Process Mining}

The necessity of privacy-preserving process mining, due to legal developments such as the GDPR, was recently discussed in $[18,19]$. In general, two approaches have been established [11]: (i) anonymizing the event data to apply standard techniques to it, and (ii) directly incorporating privacy considerations in process mining techniques. The anonymization of event logs from one organization may be done using algorithms, like PRETSA [12], that provide privacy guarantees such as $k$-anonymity [23]. These notions, based on data similarity, are widely adopted and offer protection against certain attacks like the disclosure of the identity of individuals involved in the dataset. Approaches based on cryptography $[10,20]$ have also been proposed. Following the idea to incorporate privacy guarantees directly in process mining techniques, algorithms for privacy-preserving process discovery $[17,24]$ have been proposed. Recently, techniques for privacy-preserving process mining, following either of the aforementioned paradigms, have been made available for a large audience with the tool ELPaaS [5].

All of the above techniques, with the exception of [24], concern process mining for a single organization and have not yet been adopted or evaluated for an 
inter-organizational setting. While the focus of [24] is on an inter-organizational setting, the approach targets solely the creation of a process model, while we aim at answering a wide range of analysis queries about business processes.

\subsection{Inter-organizational Process Mining}

The problem of automated discovery of process models in an inter-organizational setting has been considered in $[21,26]$. However, these approaches do not address privacy concerns. Similarly, another line of related research proposes techniques to compare executions of the same process across multiple organizations $[2,9]$, but without considering privacy requirements.

The problem of ensuring privacy in inter-organizational process mining has been addressed by Liu et al. [16]. They provide a process mining framework based on the assumption that the parties in the process are willing to share confidential information with a third (trusted) party. This assumption is unrealistic in many situations. In this paper, we address the problem of inter-organizational process mining in the context where the parties in the process are unwilling to share any execution data with each other or with a third party. In one of the embodiments of our proposal, a third party is involved for computation purposes, but this third party does not get access to any information during this computation.

\subsection{Secure Multi-party Computation}

Secure Multi-party Computation (MPC) [13] is a cryptographic functionality that allows $n$ parties to cooperatively evaluate $\left(y_{1}, \ldots, y_{n}\right)=f\left(x_{1}, \ldots, x_{n}\right)$ for some function $f$, with the $i$-th party contributing the input $x_{i}$ and learning the output $y_{i}$, and no party or an allowed coalition of parties learning nothing besides their own inputs and outputs. There exist a few approaches for constructing MPC protocols. Homomorphic secret sharing [22] is a common basis for MPC protocols. In such protocols, the arithmetic or Boolean circuit representing $f$ is evaluated gate-by-gate, constructing secret-shared outputs of gates from their secret-shared inputs. Each evaluation requires some communication between parties (except for addition gates), hence the depth of the circuit determines the round complexity of the protocol. On the other hand, there exist protocols with low communication complexity [3], allowing the secure computation of quite complex functions $f$, as long as the circuit implementing it has a low multiplicative depth.

The complexity of MPC protocols is dependent on the number of parties jointly performing the computations. Hence the typical deployment of MPC has a small number of compute nodes, also known as computation parties, which execute the protocols for evaluating gates, while an unbounded number of parties may contribute the inputs and/or receive the outputs of the computation. Several frameworks support such deployments of MPC and provide APIs to simplify the development of privacy-preserving applications [4]. One of such frameworks is Sharemind [7], whose main protocol set is based on secret-sharing among 
three computing parties. In this paper, we build on top of Sharemind, but our techniques are also applicable to other secret sharing-based MPC systems.

In Sharemind, a party can play different roles: an input party, a computation party, and/or an output party. In the case where only two parties are involved in an inter-organizational process, these two parties play the role of input parties and also that of computing parties. To fulfill the requirements of Sharemind, they need to enroll a third computing node, which merely performs computations using secret shares from which it can infer no information. ${ }^{3}$

The Sharemind framework provides its own programming language, namely the SecreC language [6], for programming privacy-preserving applications. SecreC allows us to abstract away certain details of cryptographic protocols.

\section{Multi-party Computation Based Process Mining}

This section introduces our techniques for process mining based on secure multiparty computation. Section 3.1 first clarifies our model for inter-organizational process mining including the required input data and the obtained analysis results. We then introduce our architecture for realizing the respective analysis using secure multi-party computation in Sect. 3.2. In Sect.3.3, we elaborate on vectorization and parallelization to improve the efficiency of our approach.

\subsection{Model for Inter-organizational Process Mining}

We consider a model in which an event $\log L=\left\{e_{1}, \ldots, e_{n}\right\}$ is defined as a set of events $e=(i, a, t s)$, each capturing a single execution of an activity $a$ at time $t s$, as part of a single instance $i$ of the business process. Grouping events by the latter and ordering them according to their timestamp enables the construction of traces $t=\left\langle e_{1}, \ldots, e_{m}\right\rangle$, i.e., single executions of the process, so that we refer to $i$ also as the trace identifier.

For an inter-organizational business process, an event log that records the process execution from start to end is commonly not available. Rather, different parties record sub-logs, built of events that denote activity executions at the respective party. To keep the notation concise, we consider a setting in which two parties, $I_{a}$ and $I_{b}$, execute an inter-organizational process, e.g., the airport and the airline in our motivating example. Then, each of the two parties records an event log, denoted by $L_{a}$ and $L_{b}$. Each of these logs is the projection of $L$ on the events that denote activity executions at the respective parties $I_{a}$ and $I_{b}$. We assume that each activity can only be executed by one of the parties, so that this projection is defined unambiguously.

For the above setting, we consider the scenario that the parties $I_{a}$ and $I_{b}$ want to answer some analysis queries $Q$ over the inter-organizational event log $L$, yet without sharing their $\operatorname{logs} L_{a}$ and $L_{b}$ with each other. More specifically, we focus on analysis queries that can be answered on a frequency or time-annotated

\footnotetext{
${ }^{3}$ When three or more parties are involved in a process, no external party is required.
} 
DFG of the inter-organizational process. The basic DFG captures the frequencies with which the executions of two activities have been observed to directly follow each other in a trace. Moreover, we consider temporal annotations of the directlyfollows dependencies in terms of time between the respective activity executions. Queries over the frequency and time-annotated DFGs allow us to analyze the main paths of the process, the rarely executed paths, as well as the activities that most contribute to delays in a process. Note though that only query answers are to be revealed whereas the actual DFG shall be kept private.

Formally, the time-annotated DFG is captured by an $|A| \times|A|$ matrix, where $A$ is the set of all possible activities of the process. Each cell contains a tuple $(c, \Delta)$. The counter $c$ represents the frequency with which a directly-follows dependency has been observed in $L$, i.e., for the cell $\left(a_{1}, a_{2}\right)$ it is the number of times that two events $e_{1}=\left(i_{1}, a_{1}, t s_{1}\right)$ and $e_{2}=\left(i_{2}, a_{2}, t s_{2}\right)$ follow each other directly in some trace (i.e., $i_{1}=i_{2}$ ) of $L$. Also, $\Delta$ is the total sum of the time passed by between all occurrences of the respective events, i.e., $t s_{2}-t s_{1}$ for the above events.

In inter-organizational process mining, the above time-annotated DFG cannot be computed directly, as this would require the parties to share their sub-logs.

\subsection{MPC Architecture for Process Mining}

To enable inter-organizational process mining without requiring parties to share their event logs with each other, we propose an architecture based on secure multi-party computation (MPC). As outlined in Fig. 2, we rely on a platform for MPC (in our case Sharemind [7]) that takes the event logs of the participating parties, i.e., $L_{a}$ and $L_{b}$, as secret-shared input. Inside the MPC platform, the respective data is processed in a privacy-preserving way in order to answer analysis queries over the time-annotated DFG computed from that data. In Fig. 3, we present a running example of the processing steps of the system.

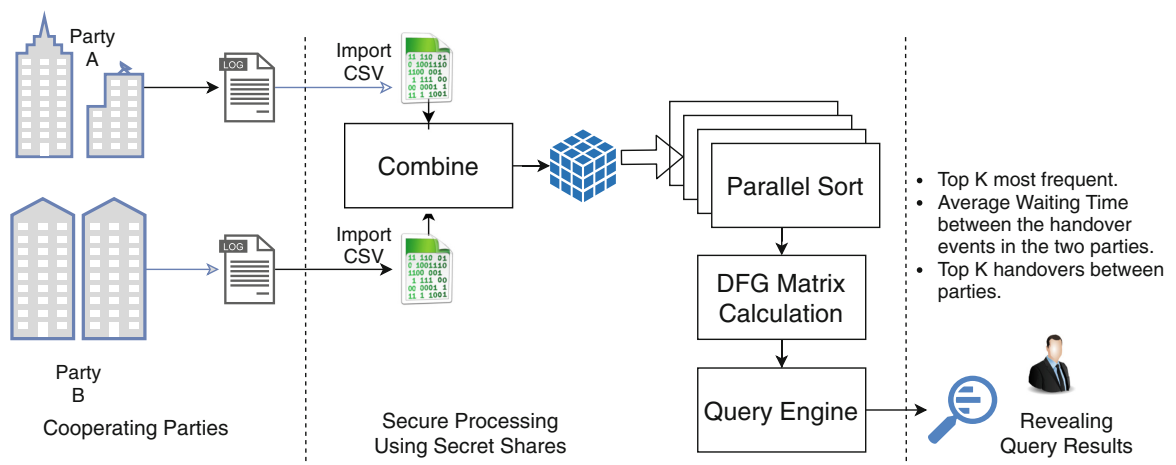

Fig. 2. Overview of the proposed approach 
Below we summarize the functionality enbodied in the proposed MPC platform for inter-organizational process mining.

Preprocessing. Each party performs the preparation of its log at its own site. The parties share the number of unique activities and the maximum number of events per trace. In Fig. 3a, we show an example with two traces. In the preprocessing step, all traces are padded to the same length, as illustrated with the blue event in Fig. 3a. The activities are transformed into a one-hot encoding that is used for masking at the DFG calculation step, as will be explained later. The logs are sorted by traces.

Combination. The parties upload their event $\operatorname{logs} L_{a}$ and $L_{b}$ to the MPC platform in a secret-shared manner. That is, the values $(i, a, t s)$ of each event (encoded as integers) are split into shares, which do not provide any information on the original values and are stored at different nodes of the platform. This way, each party can only see the total number of records uploaded by each party, but not the particular data. Subsequently, the logs are unified, creating a single log of events $L$. The combination is performed in a manner to divide the logs into processing chunks. As long as we are making the number of events per trace is fixed, that is possible by dividing the index by the number of traces for each event and assigning data from the same trace to the same chunk. In Fig. 3a, the system processes one trace with its own chunk.

Sorting. To calculate the annotated DFG, we have to determine which events follow each other in a trace by grouping the events by their trace identifier and ordering them by their timestamp. Since the trace identifier is secret-shared, we cannot group events directly. Instead, we use a privacy-preserving quicksort algorithm [14] as implemented in Sharemind to sort the events by their trace identifier. Applying the same algorithm also to the secret-shared timestamps ensures that the events of the same trace follow each other in the order of their timestamps, which is illustrated as the last step in Fig. 3a.

DFG Matrix Calculation. Next, we construct the DFG matrix inside the MPC platform, keeping it secret. Since the information on the activity of an event is secret-shared, we cannot simply process the events of traces sequentially as the matrix cell to update would not be known. Hence, we adopt a one-hot encoding for activities, so that each possible activity is represented by a binary vector of length $|A|$. To mask the actual number of possible activities, the set over which the vector is defined may further include some padding, i.e., the vector length can be chosen to be larger than $|A|$. Now, if we compute the outer product of such vectors for activities $a_{1}$ and $a_{2}$, we get a mask matrix $M$ such that $M\left[a_{1}, a_{2}\right]=1$, while all other entries are 0 . An example of such masks is given in Fig. 3b. The first mask represents the directly-follows dependency from activity $A$ to $B$ of our running example. The second mask encodes the directly-follows dependency from activity $A$ to $C$. For all sequential pairs of events in the sorted log, we sum up these matrices to get the frequency count $c$ of the directly-follows dependency 

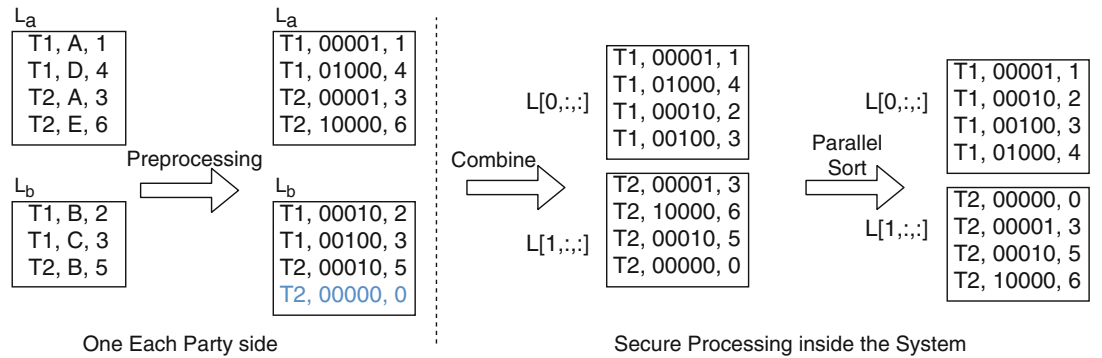

(a) Illustration of the preprocessing, combine and parallel sort steps

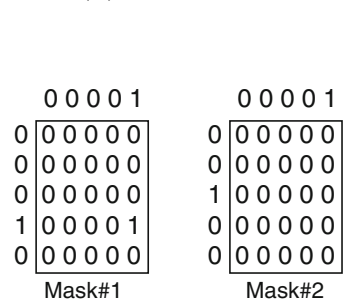

(b) Example of two masks

\begin{tabular}{|c|c|c|}
\hline Data & Shifted Data & $\begin{array}{l}\text { Same } \\
\text { Trace Flag }\end{array}$ \\
\hline $\mathrm{T} 1,00001,1$ & $\mathrm{~T} 1,00010,2$ & \\
\hline $\mathrm{T} 1,00010,2$ & $\mathrm{~T} 1,00100,3$ & \\
\hline $\mathrm{T} 1,00100,3$ & $\mathrm{~T} 1,01000,4$ & 1 \\
\hline $\mathrm{T} 1,01000,4$ & $\mathrm{~T} 2,00000,0$ & 0 \\
\hline $\mathrm{T} 2,00000,0$ & $\mathrm{~T} 2,00001,3$ & 1 \\
\hline $\mathrm{T} 2,00001,3$ & $\mathrm{~T} 2,00010,5$ & 1 \\
\hline $\begin{array}{l}\text { T2, } 00010,5 \\
\end{array}$ & $\mathrm{~T} 2,10000,6$ & 1 \\
\hline
\end{tabular}

(c) DFG Calculation using shift and a flag

\begin{tabular}{|c|c|c|c|c|c|}
\hline & A & B & C & D & E \\
\hline A & 0 & 0 & 0 & 0 & 0 \\
\hline B & 2 & 0 & 0 & 0 & 0 \\
\hline C & 0 & 1 & 0 & 0 & 0 \\
\hline D & 0 & 0 & 1 & 0 & 0 \\
\hline E & 0 & 1 & 0 & 0 & 0 \\
\hline
\end{tabular}

(d) The DFG matrix with counts

Fig. 3. Example of two event logs and their processing steps inside the system

for $\left(a_{1}, a_{2}\right)$. Multiplying $M$ by the duration between two events further enables us to derive the total sum duration passed, i.e., $\Delta$, of the directly-follows-relation. The duration operation is performed between every two consecutive events of the same trace. We can perform the duration calculation by using an elementwise vector subtraction by duplicating the dataset and then shifting its events by one as in Fig. 3c. Technically, the outer product is a function that is realized as a protocol over secret-shared data in Sharemind, and its runtime complexity is linear in $|A|[15]$.

However, the above approach could mix up events of different traces. We therefore also compute a flag $b$ that is 1 , if the trace identifiers of two events are equivalent, and 0 otherwise, which is illustrated as the "Same Trace Flag" column in Fig. 3c. Then, we multiply the mask matrix $M$ by $b$, so that the values of $M$ are ignored, if $b=0$. Again, the functionality for comparison and multiplication can be traced back to predefined protocols in Sharemind. We show the DFG matrix with counts of our running example in Fig. 3d.

Algorithm 1 summarizes the computation of the annotated DFG from the sorted, combined $\log L$, where $\llbracket \rrbracket$ denotes a secret-shared data value.

Query Answering. A query $Q$ defines a subset $S$ of the annotated DFG, which is generated by the MPC platform and revealed to the participating parties. Through sharing the $S$ solely, but not the complete annotated DFG, we are able to limit the amount of information each party can learn about the process. As an example, consider the query to derive the average waiting time between the 


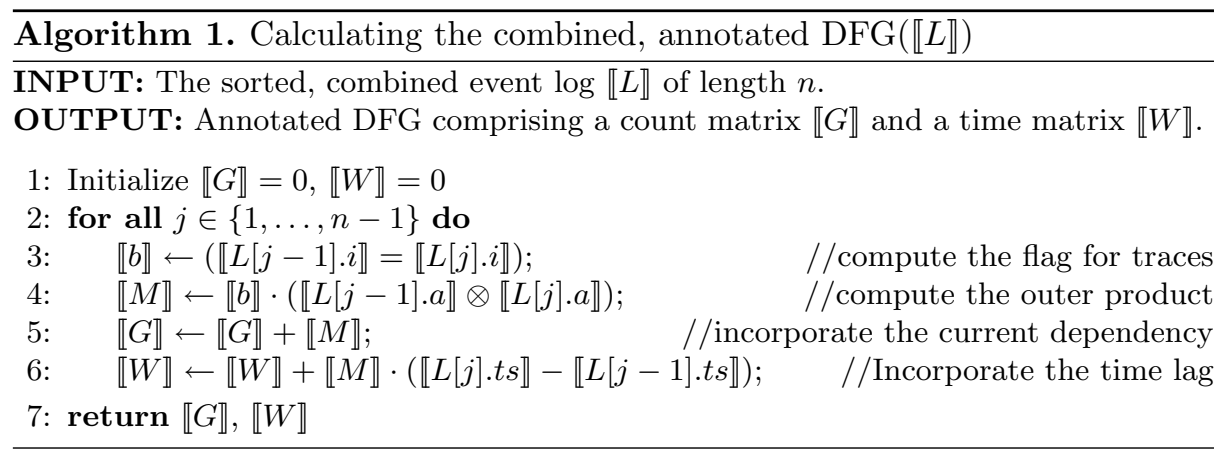

handover events between the two parties. Based on the secret-shared DFG, the respective activities may be identified through grouping and sorting the events, similar to the procedure outlined above, which is again based on the predefined protocols of an MPC platform such as Sharemind.

\subsection{Performance Optimizations}

Inter-organizational process mining using the above general architecture might suffer from scalability issues. The reason is that privacy-preserving computation through protocols over secret-shared data is inevitably less efficient than plain computation. Hence, even for functions that have a generally low runtime complexity $\left(\mathcal{O}(n)\right.$ for the combination, $\mathcal{O}(n \log (n))$ for the sorting, $\mathcal{O}\left(n m^{2}\right)$ for the calculation of the annotated DFG, where $n$ is the log length and $m$ is the number of activities), there is a non-negligible overhead induced by MPC. For instance, a naive realization of the quicksort algorithm to sort events would require $\mathcal{O}(n \log (n))$ rounds of communication between the nodes and $\mathcal{O}(n \log (n))$ value comparisons per round [14]. We therefore consider two angles to improve the efficiency of the analysis, namely vectorization and parallelization.

Vectorization. A computation that adopts a single-instruction multiple-data (SIMD) approach is highly recommended in MPC applications. Since MPC assumes continuous interaction between distributed nodes, the number of communication rounds shall be reduced as much as possible. For instance, while computing $n$ multiplications sequentially would result in $n$ rounds of communication, one may alternatively multiply element-wise two vectors of length $n$, for which one round of network communication is sufficient. Sharemind offers efficient protocols for such vector-based functions [15].

Parallelization. Further runtime improvements are obtained by parallelizing the algorithm itself. Again, our goal is to reduce the number of rounds of communication among the nodes of the MPC platform. We, therefore, split the input data into chunks, such that all chunks can be processed independently from each other. In our scenario, this is done by grouping the party logs by trace, or by a 
group of traces, generating an annotated DFG per group, and finally integrating the different DFGs. Since events of the same trace will never occur in different chunks, instead of sorting one $\log$ of length $n$, we will need to sort $c$ chunks of length $n / c$ each. Since the communication complexity of a privacy-preserving quicksort is $O(n \cdot \log n)$ [14], this improves efficiency.

The above approach raises the question of determining the size of the chunks. Separating each trace reveals the total number of events of that trace provided by a party, which may be critical from a privacy perspective. On the other hand, a small chunk size reduces the overhead of sorting. This leads to a trade-off between runtime performance and privacy considerations.

However, in our current implementation, all chunks must have the same length, as Sharemind allows parallel sorting only for equal-length vectors. Therefore, we apply padding to the traces in the log, adding dummy events (for which an empty vector in the one-hot encoding represents the activity so that the events are ignored for the DFG calculation) until the number of events of the longest trace is reached. Such padding may be employed locally, by each party, and also has the benefit that the length of individual traces is not revealed.

\section{Evaluation}

We implemented the proposed approach on top of the Sharemind multi-party computation platform. ${ }^{4}$ The source code of our implementation is available at https://github.com/Elkoumy/shareprom. The implementation is written using the SecreC programming language supported by Sharemind.

Using this implementation, we conducted feasibility and scalability experiments, specifically to address the following research questions:

RQ1: How do the characteristics of the input event logs influence the performance of the secure multi-party computation of the DFG?

RQ2: What is the effect of increasing the number of parallel chunks on the performance of the multi-party computation of the DFG?

\subsection{Datasets}

The proposed approach is designed to compute the DFG of an interorganizational process where the event log is distributed across multiple parties, and each party is responsible for executing a subset of the activities (i.e. event types) of the process. We are not aware of publicly available real-life datasets with this property. We identified a collection of synthetic inter-organizational business process event logs [8]. However, these logs are too small to allow us to conduct performance experiments (a few dozen traces per log). On the other hand, there is a collection of real-life event logs of intra-organizational processes comprising logs of varying sizes and characteristics ${ }^{5}$. From this collection, we selected three logs with different size and complexity (cf. Table 1):

\footnotetext{
${ }^{4}$ https://sharemind-sdk.github.io.

${ }^{5}$ https://data.4tu.nl/repository/collection:event_logs_real.
} 
BPIC 2013 This event log captures incident and problem management process at an IT department of a car production company.

Credit Requirement This event log comes from a process for background checking for the purpose of granting credit at a Dutch bank. It has a simple control-flow structure: All traces follow the same sequence of activities.

Traffic Fines This event log comes from a process to collect payment of fines from traffic law violations at a local police office in Italy.

Table 1. Event $\operatorname{logs}$ for evaluation

\begin{tabular}{l|r|r|r|l|l|c}
\hline Event Log & \# Events & \# Cases & \# Activities & \multicolumn{3}{|c}{ \# Events in Case } \\
\cline { 5 - 7 } & & & & Avg & Max & Min \\
\hline BPIC 2013 & 6,660 & 1,432 & 6 & 4.478 & 35 & 1 \\
Credit Requirement & 50,525 & 10,034 & 8 & 15 & 15 & 15 \\
Traffic Fines & 561,470 & 150,370 & 11 & 3.73 & 20 & 2 \\
\hline
\end{tabular}

To simulate an inter-organizational setting, we use a round-robin approach to assign each event type (activity) in the log to one of two parties. Hence, each party executes half of the event types.

\subsection{Experimental Setup}

To answer the above questions, we use the following performance measures:

- Runtime. We define runtime as the amount of time needed to transform the event logs of the two parties securely into an annotated DFG. We also report the throughput, the number of events processed by the system per second, to provide a complementary perspective.

- Communication Overhead. We define the communication overhead as the amount of data transferred between the computing parties during the multiparty computation. We measure this overhead as the volume of the data sent and received. The communication overhead gives insights into how much the performance of the multi-party computation would degrade if the computing nodes of the parties were distributed across a wide-area network.

We performed five runs per dataset per experiment. We report the average maximum values for latency and the average value for both throughput and communication overhead, across the five runs. We used Nethogs ${ }^{6}$ to measure the communication overhead, and we report the average value per compute node. The experiments were run in an environment with three physical servers as compute nodes with Sharemind installed on them. Each server has an AMD Processor 6276 and 192 GB RAM. The servers are connected using a 1GB Ethernet switch.

\footnotetext{
${ }^{6}$ https://github.com/raboof/nethogs.
} 
The experiments focus on the time needed to construct the annotated DFG, since it is the most sophisticated and time-consuming portion of the proposed analysis pipeline, due to the communication required between the compute nodes. Once the annotated DFG is available, stored in a secret-shared manner, the calculation of the actual queries has a lower complexity.

\section{$4.3 \quad$ Results}

Runtime Experiment. In Fig. 4a, we illustrate the observed execution time when varying the number of chunks used in the parallelization. We plot a bar for each chunk size. Each bar represents the runtime of the parallel sort in blue and the run time of the DFG calculation in orange. From Fig. 4a, we conclude that the runtime decreases with an increasing number of chunks, due to the parallel sorting of chunks. We also note that the runtime for the DFG calculation stays constant. In Fig. 4b, we report the number of processed events per second when varying the number of chunks. We find a consistent improvement for the throughput across all event logs.

Regarding RQ1, we summarise that the proportion of runtime between sorting and DFG calculation differs based on the event log characteristics. For the $\log$ with the largest number of event types, the DFG calculation makes up the most substantial proportion of the total runtime. In contrast, the proportion is significantly lower for the logs with a smaller number of event types. A possible explanation for this finding is the increasing size of the vectors required to represent each activity due to our bit-vector representation. Such increase results in more computational heavy calculations. Regarding RQ2, we conclude that the runtime decreases for event logs with an increasing number of chunks.

Communication Overhead. In Fig. 4c, we present the amount of data transferred to each server, again also varying the number of chunks. We observe that the communication overhead decreases with an increase in the number of chunks. These findings confirm our earlier findings regarding RQ2. In summary, a higher number of chunks leads to improved performance across all three measures.

Threats to Validity. The evaluation reported above has two limitations. First, the event logs used in the evaluation, while coming from real-life systems, are intraorganizational event logs, which we have split into separate logs to simulate an inter-organizational setting. It is possible that these logs do not capture the communication patterns found in inter-organizational processes. Second, the number of event logs is reduced, which limits the generalizability of the conclusions. The results suggest that the proposed technique can handle small-to-medium-sized logs, with relatively short traces, but there may be other characteristics of event logs that affect the performance of the proposed approach. 

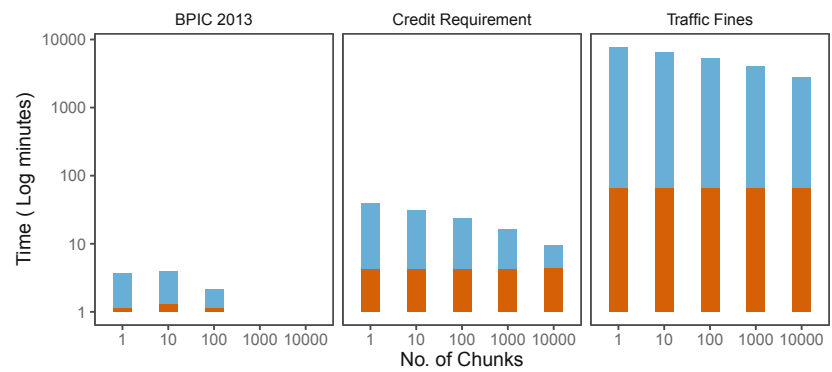

DFG calculation

Parallel Sort

(a) Runtime Experiment: Execution Time (Log) vs no. of Chunks.

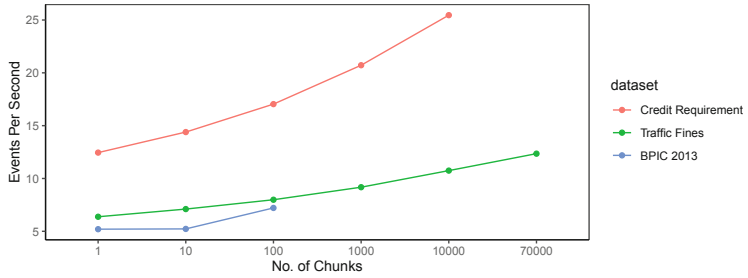

(b) Throughput Experiment: Events per Second vs no. of Chunks.
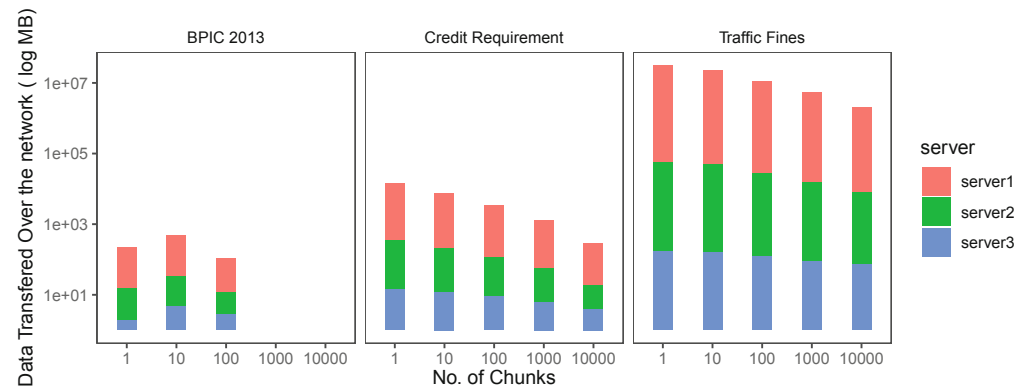

(c) Communication Overhead Experiment: Data Transferred (Log) vs no. of Chunks.

Fig. 4. Experimental evaluation of the proposed approach

\section{Conclusion}

This paper introduced a framework for inter-organizational process mining based on secure multi-party computation. The framework enables two or more parties to perform basic process mining operations over the partial logs of an interorganizational process held by each party, without any information being shared besides: (i) the output of the queries that the parties opt to disclose; and (ii) three high-level log statistics: the number of traces per log, the number of event types, and the maximum trace length. The paper specifically focuses on the computation of the DFG, annotated with frequency and temporal information. This is a basic structure used by process mining tools to perform various operations, 
including automated process discovery and various performance analysis queries (e.g. top-k bottlenecks and least-frequent and most-frequent flow dependencies).

To mitigate the high-performance overhead commonly observed for secure multi-party computation, we introduced two optimizations over the basic DFG computation algorithm: one based on vectorization of the event log and the other based on a divide-and-conquer strategy, where the log is processed in chunks.

An evaluation using real world event logs shows that with these optimizations, it is possible to compute the DFG of real-life logs with execution times that make this technique usable in practice. The divide-and-conquer approach provides opportunities to scale up the proposed technique by using a map-reduce execution-style, however not to a sufficient level to enable interactive process mining (which requires execution times in the order of seconds). Also, the approach is not able to handle logs with thousands of traces.

In future work, we will explore further optimizations to address these limitations, for example, by taking into account metadata about the event types in the event log where hand-offs occur between participants. Usually, such event types are known as they correspond to message exchange. Therefore, it becomes possible to split the logs into a "private" part and a "public" part (the latter being the points where hand-offs occur), and to process them separately using different approaches.

Another avenue for future work is to combine the proposed approach with approaches that provide complementary guarantees such as differential privacy techniques. The latter techniques allow us to noisify the DFG or the outputs from the queries of the DFG to limit the information leaked by these outputs.

Acknowledgments. This research is partly funded by ERDF via the Estonian Centre of Excellence in ICT (EXCITE) and the IT Academy programme.

\section{References}

1. van der Aalst, W.M.P.: Process Mining - Data Science in Action, Second edn. Springer, Heidelberg (2016). https://doi.org/10.1007/978-3-662-49851-4

2. Aksu, Ü., Schunselaar, D.M., Reijers, H.A.: A cross-organizational process mining framework for obtaining insights from software products: accurate comparison challenges. In: 2016 IEEE 18th Conference on Business Informatics (CBI), vol. 1, pp. 153-162. IEEE (2016)

3. Araki, T., Furukawa, J., Lindell, Y., Nof, A., Ohara, K.: High-throughput semihonest secure three-party computation with an honest majority. In: Proceedings of the 2016 ACM SIGSAC Conference on Computer and Communications Security, Vienna, Austria, 24-28 October 2016, pp. 805-817 (2016)

4. Archer, D.W., et al.: From keys to databases-real-world applications of secure multi-party computation. Comput. J. 61(12), 1749-1771 (2018)

5. Bauer, M., Fahrenkrog-Petersen, S.A., Koschmider, A., Mannhardt, F., van der Aa, H., Weidlich, M.: Elpaas: event log privacy as a service. In: Proceedings of the Dissertation Award, Doctoral Consortium, and Demonstration Track at BPM 2019 co-located with 17th International Conference on Business Process Management, BPM 2019, Vienna, Austria, 1-6 September 2019, pp. 159-163 (2019) 
6. Bogdanov, D., Laud, P., Randmets, J.: Domain-polymorphic programming of privacy-preserving applications. In: Proceedings of the Ninth Workshop on Programming Languages and Analysis for Security, p. 53. ACM (2014)

7. Bogdanov, D., Laur, S., Willemson, J.: Sharemind: a framework for fast privacypreserving computations. In: Jajodia, S., Lopez, J. (eds.) ESORICS 2008. LNCS, vol. 5283, pp. 192-206. Springer, Heidelberg (2008). https://doi.org/10.1007/9783-540-88313-5_13

8. Borkowski, M., Fdhila, W., Nardelli, M., Rinderle-Ma, S., Schulte, S.: Event-based failure prediction in distributed business processes. Inf. Syst. (2017)

9. Buijs, J.C., van Dongen, B.F., van der Aalst, W.M.: Towards cross-organizational process mining in collections of process models and their executions. In: Daniel, F., Barkaoui, K., Dustdar, S. (eds.) International Conference on Business Process Management, pp. 2-13. Springer, Heidelberg (2011). https://doi.org/10.1007/9783-642-28115-0_2

10. Burattin, A., Conti, M., Turato, D.: Toward an anonymous process mining. In: 2015 3rd International Conference on Future Internet of Things and Cloud, pp. 58-63. IEEE (2015)

11. Fahrenkrog-Petersen, S.A.: Providing privacy guarantees in process mining. In: (CAiSE Doctoral Consortium 2019), Rome, Italy, 3-7 June 2019, pp. 23-30 (2019)

12. Fahrenkrog-Petersen, S.A., van der Aa, H., Weidlich, M.: PRETSA: event log sanitization for privacy-aware process discovery. In: International Conference on Process Mining, ICPM 2019, Aachen, Germany, 24-26 June 2019, pp. 1-8 (2019)

13. Goldreich, O., Micali, S., Wigderson, A.: How to play any mental game or a completeness theorem for protocols with honest majority. In: Aho, A.V. (ed.) Proceedings of the 19th Annual ACM Symposium on Theory of Computing, pp. 218-229. ACM (1987)

14. Hamada, K., Kikuchi, R., Ikarashi, D., Chida, K., Takahashi, K.: Practically efficient multi-party sorting protocols from comparison sort algorithms. In: Kwon, T., Lee, M.-K., Kwon, D. (eds.) ICISC 2012. LNCS, vol. 7839, pp. 202-216. Springer, Heidelberg (2013). https://doi.org/10.1007/978-3-642-37682-5_15

15. Laud, P., Pankova, A.: Privacy-preserving frequent itemset mining for sparse and dense data. In: Lipmaa, H., Mitrokotsa, A., Matulevičius, R. (eds.) NordSec 2017. LNCS, vol. 10674, pp. 139-155. Springer, Cham (2017). https://doi.org/10.1007/ 978-3-319-70290-2_9

16. Liu, C., Duan, H., Zeng, Q., Zhou, M., Lu, F., Cheng, J.: Towards comprehensive support for privacy preservation cross-organization business process mining. IEEE Trans. Serv. Comput. 12(4), 639-653 (2019)

17. Mannhardt, F., Koschmider, A., Baracaldo, N., Weidlich, M., Michael, J.: Privacypreserving process mining - differential privacy for event logs. Bus. Inf. Syst. Eng. 61(5), 595-614 (2019)

18. Mannhardt, F., Petersen, S.A., Oliveira, M.F.: Privacy challenges for process mining in human-centered industrial environments. In: 2018 14th International Conference on Intelligent Environments (IE), pp. 64-71. IEEE (2018)

19. Pika, A., Wynn, M.T., Budiono, S., ter Hofstede, A.H., van der Aalst, W.M., Reijers, H.A.: Towards privacy-preserving process mining in healthcare. In: Proceedings of the Workshop on Process-Oriented Data Science in Healthcare (PODS4H) (2019)

20. Rafiei, M., von Waldthausen, L., van der Aalst, W.M.P.: Ensuring confidentiality in process mining. In: Proceedings of the 8th International Symposium on Data-driven Process Discovery and Analysis (SIMPDA 2018), Seville, Spain, 13-14 December 2018, pp. 3-17 (2018) 
21. Schulz, K.A., Orlowska, M.E.: Facilitating cross-organisational workflows with a workflow view approach. Data Knowl. Eng. 51(1), 109-147 (2004)

22. Shamir, A.: How to share a secret. Commun. ACM 22(11), 612-613 (1979)

23. Sweeney, L.: k-anonymity: a model for protecting privacy. Int. J. Uncertainty Fuzziness Knowl.-Based Syst. 10(05), 557-570 (2002)

24. Tillem, G., Erkin, Z., Lagendijk, R.L.: Mining encrypted software logs using alpha algorithm. In: SECRYPT, pp. 267-274 (2017)

25. Yao, A.C.: Protocols for secure computations. In: 23rd Annual Symposium on Foundations of Computer Science (SFCS 1982), pp. 160-164. IEEE (1982)

26. Zeng, Q., Sun, S.X., Duan, H., Liu, C., Wang, H.: Cross-organizational collaborative workflow mining from a multi-source log. Decis. Support Syst. 54(3), 1280$1301(2013)$ 